\title{
Successful onco-testicular sperm extraction from a testicular cancer patient with a single testis and azoospermia
}

\author{
Shinnosuke Kuroda', Takuya Kondo' ', Kohei Mori', Kengo Yasuda', Takuo Asai', Hiroyuki Sanjo' , Hiroyuki Yakanaka', \\ Teppei Takeshima' ${ }^{1}$, Takashi Kawahara ${ }^{2}$, Yoshitake Kato ${ }^{1}$, Yasuhide Miyoshi ${ }^{2}$, Hiroji Uemura ${ }^{2}$, Akira Iwasaki', Yasushi Yumura' \\ ${ }^{1}$ Department of Urology, Reproduction Center, Yokohama City University Medical Center, Yokohama; ${ }^{2}$ Department of Urology and Renal Transplantation, \\ Yokohama City University Medical Center, Yokohama, Japan
}

Onco-testicular sperm extraction is used to preserve fertility in patients with bilateral testicular tumors and azoospermia. We report the case of a testicular tumor in the solitary testis of a patient who had previously undergone successful contralateral orchiectomy and whose sperm was preserved by onco-testicular sperm extraction. A 35-year-old patient presented with swelling of his right scrotum that had lasted for 1 month. His medical history included a contralateral orchiectomy during childhood. Ultrasonography revealed a mosaic echoic area in his scrotum, suggesting a testicular tumor. The lesion was palpated within the normal testicular tissue along its edge and semen analysis showed azoospermia. Radical inguinal orchiectomy and onco-testicular sperm extraction were performed simultaneously. Motile spermatozoa were extracted from normal seminiferous tubules under microscopy and were frozen. Eventual intracytoplasmic sperm injection using the frozen spermatozoa is planned. Onco-testicular sperm extraction is an important fertility preservation method in patients with bilateral testicular tumors or a history of a previous contralateral orchiectomy.

Keywords: Male infertility; Testicular neoplasms; Testicular sperm retrieval

\section{Introduction}

Infertility has been reported in $50 \%-70 \%$ of patients with unilateral testicular tumors, of whom $15 \%$ exhibit azoospermia [1]. Fertilitypreserving treatment of these patients is important, particularly before adjuvant chemotherapy, since testicular tumors often develop in males younger than 5 years of age or those who are in their 20s and 30s [2]. Similarly, bilateral testicular cancer patients and testicular cancer patients with a solitary testis due to a previous contralateral orchiectomy become infertile due to the orchiectomy technique it-

Received: Oct 10, 2017 · Revised: Dec 24, 2017· Accepted: Jan 23, 2018

Corresponding author: Shinnosuke Kuroda

Department of Urology, Reproduction Center, Yokohama City University Medical Center, 4-57 Urafune-cho, Minami-ku, Yokohama, Kanagawa, Japan

Tel:+81-045-261-5656 Fax: +81-045-231-1846 E-mail: shinnosuke_1014@yahoo.co.jp

This is an Open Access article distributed under the terms of the Creative Commons Attribution Non-Commercial License (http://creativecommons.org/licenses/by-nc/4.0/) which permits unrestricted non-commercial use, distribution, and reproduction in any medium, provided the original work is properly cited. self. Therefore, fertility preservation prior to surgery is important in these patients. Herein, we report the case of a testicular tumor in the solitary testis of a patient who had previously undergone successful contralateral orchiectomy and whose spermatozoa were preserved using radical inguinal orchiectomy and simultaneous onco-testicular sperm extraction (onco-TESE).

\section{Case report}

A 35-year-old man complaining of right scrotum enlargement visited the urology department at a nearby hospital. He had a history of left orchiectomy during childhood although the cause was unknown. His wife was 26 years old and had no gynecological abnormality. During his visit, he was diagnosed with a right testicular tumor, and was expected to undergo left orchiectomy at the same hospital. Before surgery, the patient visited Reproduction Center of Yokohama City University Medical Center for sperm cryopreserva- 


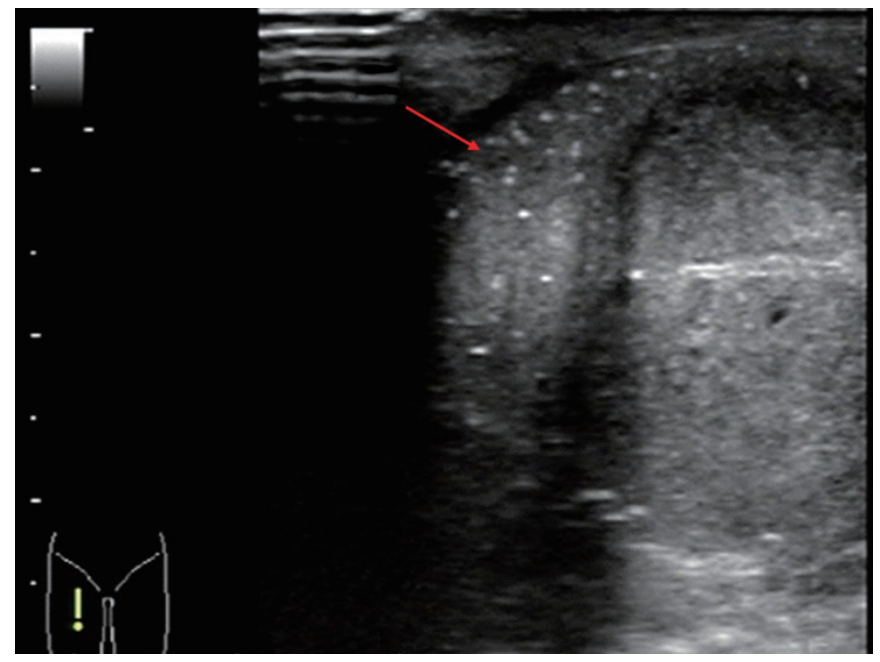

Figure 1. Ultrasonography of the right testis. Normal testicular tissue was palpated along the edge of the testis (arrow).

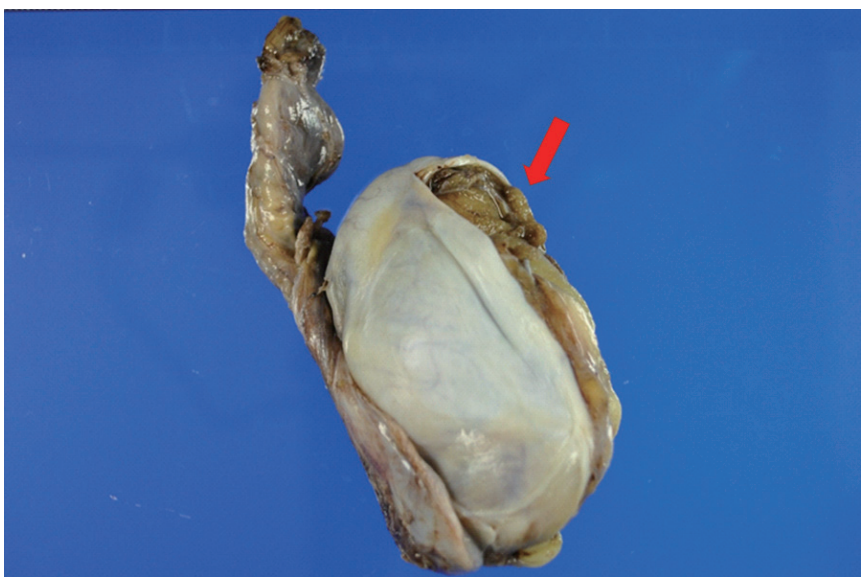

Figure 2. Formalin-fixed specimen extracted from the right testis. Although most of the testis was occupied by the tumor, a small amount of normal seminiferous tubules was detected (arrow).

tion. Written informed consent was obtained for research purposes at the initial visit. The right scrotum was swollen to the size of a chicken egg without pain. A laboratory examination showed almost normal levels of lactate dehydrogenase, $\alpha$-fetoprotein, and $\beta$-human chorionic gonadotropin. His testosterone level was low $(1.96 \mathrm{ng} / \mathrm{mL})$. Ultrasonography indicated that most of the testis exhibited a mosaic echoic pattern. However, normal testicular tissue was palpated along the edge of the testis (Figure 1). Semen analysis revealed azoospermia, and no spermatozoa were observed in the pellet after centrifugal separation. After discussions with the referring hospital, semiemergency left radical inguinal orchiectomy and onco-TESE were performed simultaneously at our reproductive medicine center. Although most of the testicular space was occupied by the tumor, a small amount of normal seminiferous tubules was detected and ex-

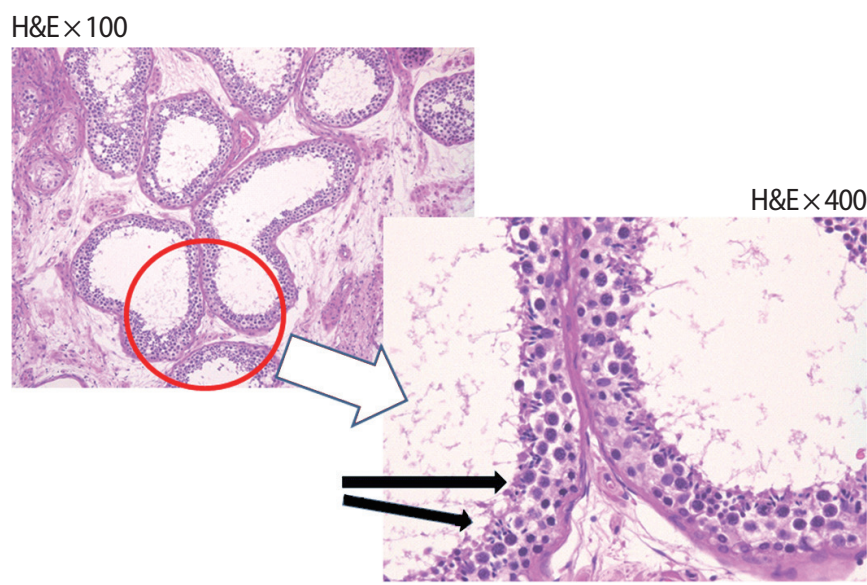

Figure 3. Pathological analysis of the normal testicular tissue. Normal spermatogenesis was confirmed in some seminal tubules (black arrows). The mean Johnsen score count was 8.3.

tracted macroscopically (Figure 2). Ex vivo sperm extraction under a microscope during bench surgery was performed from the extracted specimen. Motile spermatozoa were detected and approximately 15,600 spermatozoa were obtained. They were divided into 10 vials for cryopreservation. Pathological analysis of the tumor lesion revealed many atypical cells with clear cell bodies and conspicuous nucleoli, thereby indicating a typical seminoma. The diagnosis was pure seminoma, pT1NOMO. Normal spermatogenesis was confirmed in a portion of the normal tissue and the mean Johnsen score count was 8.3 (Figure 3). There was no tumor recurrence at a 6-month follow-up visit, and the patient currently receives ongoing testosterone supplementation due to depressed testosterone levels after surgery.

\section{Discussion}

Over the past decade, the treatment of patients with testicular cancer has improved dramatically with the development of anticancer drugs, including platinum formulations [3]. Yet, as the number of young cancer survivors continues to increase, there must be a concomitant improvement of the quality of the life of these patients [4]. Furthermore, fertility preservation among adolescent and young adult patients is critical, with gonadotropic therapy being a critical form of treatment. If spermatozoa viability is confirmed prior to cancer therapy, cryopreservation of semen should always be recommended before performing treatment that can result in infertility, such as radiotherapy, chemotherapy, or surgery [5]. Furthermore, onco-TESE should be considered for patients whose semen analysis indicates azoospermia.

Onco-TESE is a procedure that allows spermatozoa to be obtained from the normal testis of patients who do not emit sperm prior to cancer therapy [6]. Onco-TESE can be used in patients with azoosper- 
Table 1. Previous cases of patients with testicular cancer who underwent orchiectomy and onco-TESE in a single testis

\begin{tabular}{lclccccc}
\hline Study & Age (yr) & \multicolumn{1}{c}{ Past history } & $\begin{array}{c}\text { Tumor } \\
\text { size }(\mathrm{mm})\end{array}$ & Sperm analysis & $\begin{array}{c}\text { Sperm } \\
\text { retrieval }\end{array}$ & Pregnancy & Live birth \\
\hline Descombe et al. (2008) [10] & 33 & Torsion of spermatic cord & 40 & Severe oligozoospermia & 0 & - \\
& 28 & Torsion of spermatic cord & - & Azoospermia & 0 & 0 \\
Furuhashi et al. (2013) [8] & 28 & Testicular tumor & 6 & Azoospermia & 0 & 0 & - \\
& 36 & Testicular tumor & 30 & Severe oligozoospermia & 0 & $\times$ \\
& 47 & Testicular tumor & 40 & Azoospermia & $\times$ & $\times$ \\
Current study & 32 & Unknown & 70 & Azoospermia & 0 & - & $\times$ \\
\hline
\end{tabular}

Onco-TESE, onco-testicular sperm extraction.

mia, severe oligozoospermia, or an ejaculation disorder before chemotherapy, as well as in patients with a single testis whose fertility would be damaged by testicular cancer treatment.

To our knowledge, there have been reports of only 44 patients, including our patient, who have undergone onco-TESE [6-10]. In these reports, spermatozoa were successfully preserved in only 23 cases (52.2\%). Moreover, only six cases of onco-TESE, including this case, have been reported in patients with a single testis after unilateral orchiectomy (Table 1). In five of the six cases, sperm retrieval was successful. Descombe et al. [10] reported a live twin birth after intracytoplasmic sperm injection using sperm retrieved by onco-TESE. Previous studies have reported an inverse correlation between tumor size and the presence of spermatogenesis, with $81 \%-86 \%$ and $57 \%$ of patients with a tumor size of $1-2 \mathrm{~cm}$ and $5 \mathrm{~cm}$, respectively, exhibiting healthy spermatozoa production [11]. Similarly, Suzuki et al. [12] showed a negative correlation between the Johnsen score count and the maximum diameter of the tumor. They further found that spermatogenesis improved in cases with a greater distance between the seminiferous tubules and the tumor. Compared with the other previous five cases of onco-TESE in patients with a single testis, the tumor in our patient was the largest. In addition, while there was extensive normal testicular tissue apart from the tumor lesion in the other five cases, the tumor cells occupied most of the testis in our case, and very little normal tissue was confirmed. This is the first report of successful onco-TESE from a single testis occupied by a large tumor with very few normal seminiferous tubules. Although we first speculated that, given our initial findings, testicular sperm extraction would be difficult, we were able to retrieve and store spermatozoa successfully. In cases such as the one presented herein, the surgeon should attempt to identify healthy seminiferous tubules within the tumor tissue. Further study of such cases and the associated fertility outcomes should be conducted.

In conclusion, we experienced a case of azoospermia in a patient with a large testicular tumor in a single testis. We successfully performed sperm retrieval through onco-TESE. This is the first report of successful onco-TESE from a single testis occupied by a large tumor with very few normal seminiferous tubules. In such cases, it is important to search for normal tissue within the testicle.

\section{Conflict of interest}

No potential conflict of interest relevant to this article was reported.

\section{References}

1. Safsaf A, Sibert L, Cleret JM, Perdrix A, Milazzo JP, Gobet F, et al. Concomitant unilateral and synchronous bilateral testis cancer in azoospermic dizygotic twins: differential management of fertility preservation. Fertil Steril 2011;95:2434.e11-3.

2. Albers P, Albrecht W, Algaba F, Bokemeyer C, Cohn-Cedermark G, Fizazi K, et al. Guidelines on testicular cancer: 2015 update. Eur Urol 2015;68:1054-68.

3. Einhorn LH. Testicular cancer as a model for a curable neoplasm: the Richard and Hinda Rosenthal Foundation Award lecture. Cancer Res 1981;41(9 Pt 1):3275-80.

4. Ginsberg JP. Educational paper: the effect of cancer therapy on fertility, the assessment of fertility and fertility preservation options for pediatric patients. Eur J Pediatr 2011;170:703-8.

5. Loren AW, Mangu PB, Beck LN, Brennan L, Magdalinski AJ, Partridge $\mathrm{AH}$, et al. Fertility preservation for patients with cancer: American Society of Clinical Oncology clinical practice guideline update. J Clin Oncol 2013;31:2500-10.

6. Schrader M, Muller M, Sofikitis N, Straub B, Krause H, Miller K. "Onco-tese": testicular sperm extraction in azoospermic cancer patients before chemotherapy-new guidelines? Urology 2003; 61:421-5.

7. Tsutsumi S, Kawahara T, Takeshima T, Chiba S, Uemura K, Otani M, et al. Onco-testicular sperm extraction (onco-TESE) for bilateral testicular tumors: two case reports. J Med Case Rep 2017;11:139.

8. Furuhashi K, Ishikawa T, Hashimoto H, Yamada S, Ogata S, Mizusawa Y, et al. Onco-testicular sperm extraction: testicular sperm extraction in azoospermic and very severely oligozoospermic 
cancer patients. Andrologia 2013;45:107-10.

9. Roque M, Sampaio M, Salles PG, Geber S. Onco-testicular sperm extraction: birth of a healthy baby after fertility preservation in synchronous bilateral testicular cancer and azoospermia. Andrologia 2015;47:482-5.

10. Descombe L, Chauleur C, Gentil-Perret A, Aknin-Seifer I, Tostain J, Levy R. Testicular sperm extraction in a single cancerous testicle in patients with azoospermia: a case report. Fertil Steril 2008;90: 443.e1-4.

11. Choy JT, Wiser HJ, Bell SW, Cashy J, Brannigan RE, Kohler TS. Predictors of spermatogenesis in orchiectomy specimens. Urology 2013;81:288-92.

12. Suzuki K, Shin T, Shimomura Y, Iwahata T, Okada H. Spermatogenesis in tumor-bearing testes in germ cell testicular cancer patients. Hum Reprod 2015;30:2853-8. 